\title{
Symptom Burden Among Cancer Survivors: Impact of Age and Comorbidity
}

\author{
Jun J. Mao, MD, MSCE, Katrina Armstrong, MD, MSCE, Marjorie A. Bowman, MD, MPA, \\ Sharon X. Xie, PhD, Rachel Kadakia, BA, and John T. Farrar, MD, PhD
}

Background: Previous research among specific cancer populations has shown high but variable symptom burden; however, very little is known about its extent and pattern among the entire population of US cancer survivors, which is more clinically relevant to primary care physicians.

Methods: To determine the prevalence of ongoing symptom burden among cancer survivors and compare it with the general population without cancer, we analyzed data from the 2002 National Health Interview Survey, which included 1,904 cancer survivors and 29,092 controls. Main outcome measures included self-reported ongoing pain, psychological distress, and insomnia. Multivariate logistic regression models were used to adjust for confounders and test for interactions.

Results: The rates of ongoing pain, psychological distress, and insomnia among cancer survivors were $34 \%, 26 \%$, and $30 \%$, respectively, and were significantly higher (all $P<.001$ ) than controls without a history of cancer $(18 \%, 16 \%$, and $17 \%)$. Compared with controls in the same age groups, younger survivors (younger than 50) were much more likely to report ongoing symptoms than older survivors (older than 64); adjusted odds ratios were 2.96 and 1.36 for pain in the respective age groups $(P<$ .001). Comorbidities also interact with cancer status and contribute to a marked increase in reports of ongoing symptom burden among cancer survivors, with a greater number of comorbidities leading to greater degree of symptom burden in a dose-dependent manner $(P<.001)$.

Conclusions: The symptom burden among cancer survivors on a population level is substantial and can be impacted by other comorbidities. Thus, engaging primary care physicians in the design, testing, and implementation of effective interventions is important to reduce the symptom burden among cancer survivors. (J Am Board Fam Med 2007;20:434-443.)

Each year, more than 1 million people are diagnosed with cancer in the United States. This number is expected to double by the year 2050 as a result of the increase in age and size of the US population. ${ }^{1,2}$ Cancer is often considered "the most

This article was externally peer reviewed.

Submitted 27 December 2006; revised 24 March 2007; accepted 29 March 2007.

From the Department of Family Medicine and Community Health (JJM, MAB, RK); the Center for Clinical Epidemiology and Biostatistics (JJM, KA, SXX, JTF); the Abramson Cancer Center (JJM, KA, MAB, JTF); and the Department of Medicine (KA), University of Pennsylvania School of Medicine, Philadelphia.

Funding: We thank the University of Pennsylvania Robert Wood Johnson Health and Society Scholar Program for financial support of this project. Dr. Mao is supported by a training grant from HRSA 1 D14 HP 008084.

Prior presentation: Part of the content was orally presented at the North American Primary Care Research Group Annual Meeting in Tucson, AZ, 17 October 2006. Research poster presented at the Third Biennial Cancer Survivorship Research Conference: Cancer Survivorship - Embracing the Future, October 2006, North Bethesda, MD.

Conflict of interest: none declared.

Disclaimer: Dr. Mao had full access to all of the data in the study and takes responsibility for the integrity of the data and the accuracy of the data analysis. dreaded disease" not only because of the perceived mortality but also because of the severe suffering associated with the disease and its conventional treatments, of which the most commonly reported are pain, ${ }^{3-5}$ psychological distress, ${ }^{6,7}$ and insomnia. ${ }^{8,9}$

Previous research has shown that $14 \%$ to $100 \%$ of oncology outpatients experience pain. ${ }^{3}$ The frequency of psychological distress, such as depression and anxiety, ranges from $0 \%$ to $44 \%$ in cancer patients. ${ }^{6,10,11}$ The prevalence rate for insomnia among patients with different types of cancer and measured at different courses of the disease range from $30 \%$ to $59 \% .^{8,12,13}$ These symptoms occur individually or in combination, negatively impact the patient's quality of life, and result in increased morbidity and health care costs. ${ }^{4,14-16}$

Despite the importance of symptom research in cancer, the existing literature is limited. Most pre-

Corresponding author: Jun J. Mao, MD, MSCE, Department of Family Medicine and Community Health, University of Pennsylvania School of Medicine, 3400 Spruce Street, 2 Gates Building, Philadelphia, PA 19104 (E-mail: maoj@uphs.upenn.edu). 
vious studies used small and highly selected clinical populations such as patients in specialty clinics in comprehensive cancer centers. On a population level, very little is known about the extent of symptom burden among people with a previous cancer diagnosis, especially when compared with people without cancer or with other chronic diseases. Furthermore, because early detection and treatment advances have transformed cancer into a chronic disease for many patients, people with a cancer diagnosis are increasingly likely to interact with primary care physicians in settings outside of specialty clinics in large academic centers. A population-based study is needed to understand the symptom burden among cancer survivors on a community level. Thus, the specific objectives of this study are (1) to estimate the prevalence of ongoing symptoms such as recurring pain, frequent depression and anxiety, and regular insomnia among cancer survivors in a large nationally representative sample; (2) to quantify the magnitude of symptom burden among cancer survivors by a comparison with the general population without cancer; and (3) to understand the impact of comorbidity on symptom burden among cancer survivors.

\section{Methods \\ Study Sample}

The study population was derived from all of the adults who participated in the 2002 National Health Interview Survey (NHIS), an annual multistage survey conducted in a nationally representative sample of the civilian, noninstitutionalized population of the United States. The Centers for Disease Control and Prevention's National Center for Health Statistics conducted the NHIS Survey through confidential in-person interviews. ${ }^{17}$ The survey was conducted both in English and Spanish. The data for this study was extracted from the Sample Adult Core component of the 2002 NHIS. Details on the survey design and content can be found online at the National Center for Health Statistics website. ${ }^{18}$ This study was approved by the Institutional Review Board at the University of Pennsylvania.

\section{Cancer Survivorship Status}

The status of "cancer survivor," defined as anyone who has been diagnosed with cancer from the time of diagnosis through the balance of their life, ${ }^{1}$ was determined by response to the question, Have you ever been told by a doctor or other health professional that you had cancer or a malignancy of any kind? All participating adults were asked this question. ${ }^{17}$ People who responded "yes" were then asked about the type(s) of cancer and their age at each cancer diagnosis, if there was more than one. Eight nonoverlapping cancer types were created. More than 100 patients were in each of the cancer groups labeled breast, prostate, colorectal, cervix, uterine, and melanoma. A multiple cancer group was created for people who had been diagnosed with more than one cancer. The remaining cancer types were grouped as "other." The variable of time since cancer diagnosis was calculated by subtracting the age at diagnosis from the age at the time of the survey. We created 4 categories of "time since diagnosis" (within the past year, 2 to 5 years, 6 to 10 years, and $\geq 11$ years). ${ }^{2}$ When the subtraction yielded inconsistent results (eg, negative numbers), we used the methods described by Yabroff et $\mathrm{al}^{2}$ to recode this variable.

\section{Symptom Burden Measures}

The 2002 NHIS survey contained the following items with dichotomous yes/no response options: During the past 12 months have you "... had recurring pain"; “. . . been frequently depressed or anxious"; or "... regularly had insomnia or trouble sleeping?" Responses of "yes" were defined as cases. These items, using modifiers, were designed to assess self-reported, clinically bothersome, ongoing symptom distress beyond the general symptoms that affect everyone at one time or another. ${ }^{19}$

\section{Sociodemographic Characteristics}

The $4 \mathrm{racial} / \mathrm{ethnic}$ groups we used were the same as those defined in the NHIS: Hispanic; non-Hispanic white; non-Hispanic black, and non-Hispanic other. The annual household income was categorized by NHIS as $<\$ 20,000$ and $\geq \$ 20,000$. We created 3 age categories for analysis purposes: 18 to 49,50 to 64 , and $\geq 65$. We classified education as high school graduate or less, some college or technical school, and college or more advanced degree. Marital status was categorized in 2 ways: currently married or living with a partner and not currently partnered or married. Comorbidity was measured by a series of questions related to functional limitations. Those who identified any limitations were given additional questions about the health condi- 
tions causing that limitation. Comorbid conditions were then categorized by the absolute number of conditions identified as causing limitations (ie, none, $1,2,3$ or more). ${ }^{2}$

\section{Statistical Analyses}

Bivariate analysis was performed to compare demographic and socioeconomic variables among cancer survivors and control populations. Prevalence estimates of pain, psychological distress, and insomnia were then determined. To compare symptom burden between cancer survivors and general population controls, we developed multivariate logistic regression models using dichotomous variables for symptom burden and cancer survivorship status as the primary association of interest, and adjusting for age, sex, race/ethnicity, education, and income. As part of the model development, we tested interaction among cancer survivorship status and each covariate separately, but we found that such effect modification existed only between age and cancer survivorship. We estimated the predicted probability of the experience of a given symptom stratified by cancer survivor status, age, and sex while adjusting for other covariates.

To understand the impact of comorbidity on symptom burden among cancer survivors, we developed logistic regression models with symptom burden as dichotomous dependent variables and cancer and comorbidity as interaction items. We controlled for age, sex, race/ethnicity, education, and income.

Furthermore, we explored how symptom burden may differ among cancer survivors by cancer types and time since diagnosis using logistic regression models. Symptoms were used as the dependent variables and the cancer types and time since diagnosis were used as the independent variables while adjusting for age, education, race/ethnicity, and income. Breast cancer was chosen as the reference group for cancer type because much of cancer symptom research has been conducted in the breast cancer population; thus our findings could be interpreted in the context of existing literature. Because certain cancers are sex specific, our model did not include sex as a covariate.

All statistical analyses were performed using survey analysis package from STATA (version 8.0; StataCorp, College Station, TX). We used "svy" commands to generate population estimates and make comparisons among different groups while taking into account the multistage sampling, clustering, and stratification design of the NHIS. ${ }^{20}$ All statistical tests were 2-sided.

\section{Results}

\section{Study Population Characteristics}

In 2002, 31,044 noninstitutionalized people aged 18 years or older participated in the survey, with an overall response rate of $74.3 \% .^{21}$ Of these participants, $48(0.16 \%)$ did not answer the cancer diagnosis question and were excluded from the analysis. Our final sample consisted of 1,904 cancer survivors and 29,092 general population controls. For the time since diagnosis analyses, we excluded 111 (5.8\%) patients who had incomplete data for these calculations. Table 1 reports the demographic and socioeconomic characteristics of cancer survivors and controls. In this sample of cancer survivors, 181 (9.5\%) people had more than one cancer, 326 (17.1\%) had breast cancer, 195 (10.2\%) had prostate cancer, 144 (7.6\%) had colorectal cancer, 177 (9.3\%) had cervical cancer, 107 (5.6\%) had uterine cancer, and 107 (5.6\%) had melanoma. Stratified by time since cancer diagnosis, 330 (18.4\%) patients were diagnosed with cancer in the past year, $534(29.4 \%)$ between 2 and 5 years, 335 (18.7\%) between 6 and 10 years, and $594(33.1 \%)$ more than 10 years earlier.

\section{Symptom Burden: Cancer Compared With the General Population}

Overall, $34 \%$ of cancer survivors reported recurrent pain, 26\% reported frequent depressed or anxious mood, and $30 \%$ reported regular insomnia. These rates were substantially higher than the general population without a previous cancer diagnosis (17\%, 15\%, 17\% for pain, psychological distress, and insomnia, respectively). Our analysis demonstrated an association between cancer diagnosis and increased symptom burden that differed by age. A greater difference was seen among patients younger than 50 than among those older than 64 (see Table 2). Estimates of conditional probabilities further clarified this relationship (see Figure 1). For example, among women younger than age $50,40 \%$ of cancer survivors versus $17 \%$ of the general population controls reported ongoing psychological distress, which yielded a $23 \%$ risk difference. On the other hand, for women older than age $64,25 \%$ of cancer survivors versus $18 \%$ of the general popu- 


\begin{tabular}{|c|c|c|c|}
\hline Characteristics & $\begin{array}{l}\text { Cancer Survivors } \\
\quad(\mathrm{n}=1,904)\end{array}$ & $\begin{array}{l}\text { General Population Controls } \\
\qquad(\mathrm{n}=29,092)\end{array}$ & $P^{*}$ \\
\hline \multicolumn{4}{|l|}{ Age (years) } \\
\hline$<50$ & 22.9 & 65.3 & \multirow[t]{3}{*}{$<.001$} \\
\hline 50 to 64 & 26.9 & 20.8 & \\
\hline$\geq 65$ & 50.2 & 13.9 & \\
\hline \multicolumn{4}{|l|}{ Sex } \\
\hline Male & 41.2 & 48.4 & \multirow[t]{2}{*}{$<.001$} \\
\hline Female & 58.8 & 51.6 & \\
\hline \multicolumn{4}{|l|}{ Education attainment } \\
\hline High school graduate or less & 52.4 & 46.1 & \multirow[t]{3}{*}{$<.001$} \\
\hline Some college or technical school & 27.4 & 29.2 & \\
\hline College or more advanced degree & 20.2 & 24.7 & \\
\hline \multicolumn{4}{|l|}{ Race/ethnicity } \\
\hline Hispanic & 3.8 & 11.5 & \multirow[t]{4}{*}{$<.001$} \\
\hline Non-Hispanic white & 89.0 & 72.2 & \\
\hline Non-Hispanic black & 5.4 & 11.7 & \\
\hline Other (non-Hispanic) & 1.8 & 4.6 & \\
\hline \multicolumn{4}{|l|}{ Marital status } \\
\hline Currently married/living with partner & 64.2 & 63.8 & \multirow[t]{2}{*}{0.74} \\
\hline Not currently married/partnered & 35.8 & 36.3 & \\
\hline \multicolumn{4}{|l|}{ Annual household income } \\
\hline$<\$ 20,000$ & 24.6 & 19.1 & \multirow[t]{2}{*}{$<.001$} \\
\hline$\geq \$ 20,000$ & 75.4 & 80.9 & \\
\hline \multicolumn{4}{|l|}{ Comorbid conditions } \\
\hline None & 39.2 & 76.4 & \multirow[t]{4}{*}{$<.001$} \\
\hline One & 39.1 & 18.1 & \\
\hline Two & 12.2 & 3.9 & \\
\hline Three or more & 9.5 & 1.6 & \\
\hline \multicolumn{4}{|l|}{ Time since cancer diagnosis (years) } \\
\hline 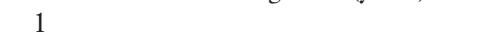 & 18.4 & & \\
\hline 2 to 5 & 29.8 & & \\
\hline 6 to 10 & 18.7 & & \\
\hline$\geq 11$ & 33.1 & & \\
\hline
\end{tabular}

Data source: National Health Interview Survey 2002; weighted percentages were extrapolated from the US census (2000) for the adult civilian. All data presented as percentages.

* Categorical variables were compared with univariate logistic regression; $P$ values are two-sided.

lation controls without cancer reported similar complaints, yielding a $7 \%$ risk difference.

Women were more likely to report ongoing symptoms independent of cancer survivorship status. The gender differences were greatest for psychological distress and insomnia and smallest for pain with $95 \%$ confidence intervals not overlapping (see Table 2). Higher levels of education and higher income were associated with lower levels of ongoing symptoms (see Table 2). For pain and insomnia, the effect becomes significant only when people acquired a college or higher education; however, with regards to psychological distress, each increase in level of education was linked to a significant decrease in symptom prevalence. Nonwhite races were significantly less likely to report ongoing symptoms except for psychological dis- tress, which Non-Hispanic blacks reported at a similar rate as whites.

\section{Impact of Comorbidity on Symptom Burden}

Our analyses demonstrated an interaction between cancer status and comorbidity that results in increased symptom burden. Both cancer status and the presence of comorbidity are independently associated with greater symptom burden. Although the specific impact of cancer on the overall symptom burden is smaller among survivors with comorbidities than those without, the overall symptom burden increases with the number of comorbidities (see Table 3; both the interaction terms and models were highly significant at $P<.001)$. Furthermore, cancer survivors reported higher symptom burden compared with noncancer controls at each level of comorbidities. 
Table 2. Symptom Burden Among Cancer Survivors Differs by Age

\begin{tabular}{|c|c|c|c|c|c|c|c|c|c|}
\hline \multirow[b]{2}{*}{ Characteristics } & \multicolumn{3}{|c|}{ Pain } & \multicolumn{3}{|c|}{ Psychological Distress } & \multicolumn{3}{|c|}{ Insomnia } \\
\hline & OR & $95 \% \mathrm{CI}$ & $P$ & OR & $95 \% \mathrm{CI}$ & $P$ & OR & $95 \% \mathrm{CI}$ & $P$ \\
\hline CS versus control (unadjusted) & 2.44 & $2.16-2.74$ & $<.001$ & 1.98 & $1.76-2.22$ & $<.001$ & 2.09 & $1.83-2.38$ & $<.001$ \\
\hline \multicolumn{10}{|l|}{ Age (years) } \\
\hline$<50$ & 2.96 & $2.28-3.85$ & $<.001$ & 2.94 & $2.35-3.67$ & $<.001$ & 2.70 & $2.09-3.48$ & $<.001$ \\
\hline 50 to 64 & 2.23 & $1.76-2.83$ & $<.001$ & 1.84 & $1.45-2.34$ & $<.001$ & 1.80 & $1.40-2.31$ & $<.001$ \\
\hline$>64$ & 1.36 & $1.14-1.63$ & .001 & 1.48 & $1.23-1.79$ & $<.001$ & 1.44 & $1.18-1.75$ & $<.001$ \\
\hline Sex (female vs male) & 1.24 & $1.15-1.32$ & $<.001$ & 1.51 & $1.39-1.64$ & $<.001$ & 1.47 & $1.37-1.59$ & $<.001$ \\
\hline \multicolumn{10}{|l|}{ Education } \\
\hline High school or less* & 1 & & & 1 & & & 1 & & \\
\hline Some college & 0.98 & $0.90-1.06$ & .56 & 0.86 & $0.79-0.94$ & .001 & 1.01 & $0.92-1.10$ & .877 \\
\hline College or more advanced & 0.73 & $0.66-0.81$ & $<.001$ & 0.57 & $0.51-0.64$ & $<.001$ & 0.70 & $0.63-0.76$ & $<.001$ \\
\hline \multicolumn{10}{|l|}{ Race/ethnicity } \\
\hline Non-Hispanic white* & 1 & & & 1 & & & 1 & & \\
\hline Non-Hispanic black & 0.84 & $0.75-0.94$ & .003 & 0.90 & $0.80-1.02$ & .102 & 0.78 & $0.70-0.88$ & $<.001$ \\
\hline Hispanic & 0.55 & $0.48-0.63$ & $<.001$ & 0.85 & $0.75-0.96$ & .007 & 0.77 & $0.69-0.87$ & $<.001$ \\
\hline Other (non-Hispanic) & 0.62 & $0.49-0.78$ & $<.001$ & 0.67 & $0.52-0.86$ & .002 & 0.57 & $0.45-0.72$ & $<.001$ \\
\hline \multicolumn{10}{|l|}{ Annual household income } \\
\hline$(<\$ 20,000$ vs. $\geq \$ 20,000)$ & 1.37 & $1.25-1.51$ & $<.001$ & 1.85 & $1.69-2.04$ & $<.001$ & 1.52 & $1.39-1.67$ & $<.001$ \\
\hline
\end{tabular}

*Models included cancer survivor status and age as interaction terms and controlled for sex, education, race/ethnicity, and income. $\mathrm{CS}$, cancer survivors; OR, odds ratio; CI, confidence interval.

\section{Symptom Burden: Differences among Subgroups of Cancer Survivors}

Controlling for time since diagnosis and sociodemographic variables, with breast cancer as the reference group, patients with a history of multiple cancers were significantly more likely to report recurring pain and psychological distress. People with a history of cervical or uterine cancer were more likely to report psychological distress and insomnia than were patients with a history of breast cancer (Table 4). After adjusting for cancer types and sociodemographic variables, cancer survivors more than 2 years from their cancer diagnosis were significantly less likely to report regular insomnia that those who were recently diagnosed; however, the report of recurring pain and frequent psychological distress did not decrease significantly (Table 4).

\section{Discussion}

Using a population-based national sample, we found that a greater proportion of cancer survivors experienced ongoing symptom burden than did people in the general population without cancer. The association between symptom burden and cancer status was found to be greater among younger than older patients. In addition, comorbidities in- teract with cancer to result in greater symptom burden among cancer survivors. With the number of cancer survivors in the United States exceeding 10 million, ${ }^{22}$ primary care physicians should be aware of the substantial symptom burden among these patients so that effective assessment and treatment can take place in the clinical setting.

Our estimated prevalence rates for ongoing symptom distresses among cancer survivors, including pain (34\%), psychological distress (26\%), and insomnia (30\%), fell into the lower range of previous research findings (14\% to $100 \%, 0 \%$ to $44 \%$, and $30 \%$ to $59 \%$ for pain, psychological distress, and insomnia, respectively). The variation among the studies may be a result of the sampling frame. ${ }^{19}$ Many earlier studies sampled subjects from specialty oncology or symptom clinics whereas our study was drawn from a population-based sample. Therefore we provided a more accurate point estimate of prevalence rate of the symptom burden among the general population of cancer survivors. The different operational definitions and psychometric measures can also introduce discrepancies in evaluating these subjective complaints. ${ }^{10,19}$ The age and sex of the population varies with each study and may further account for substantial differences in symp- 


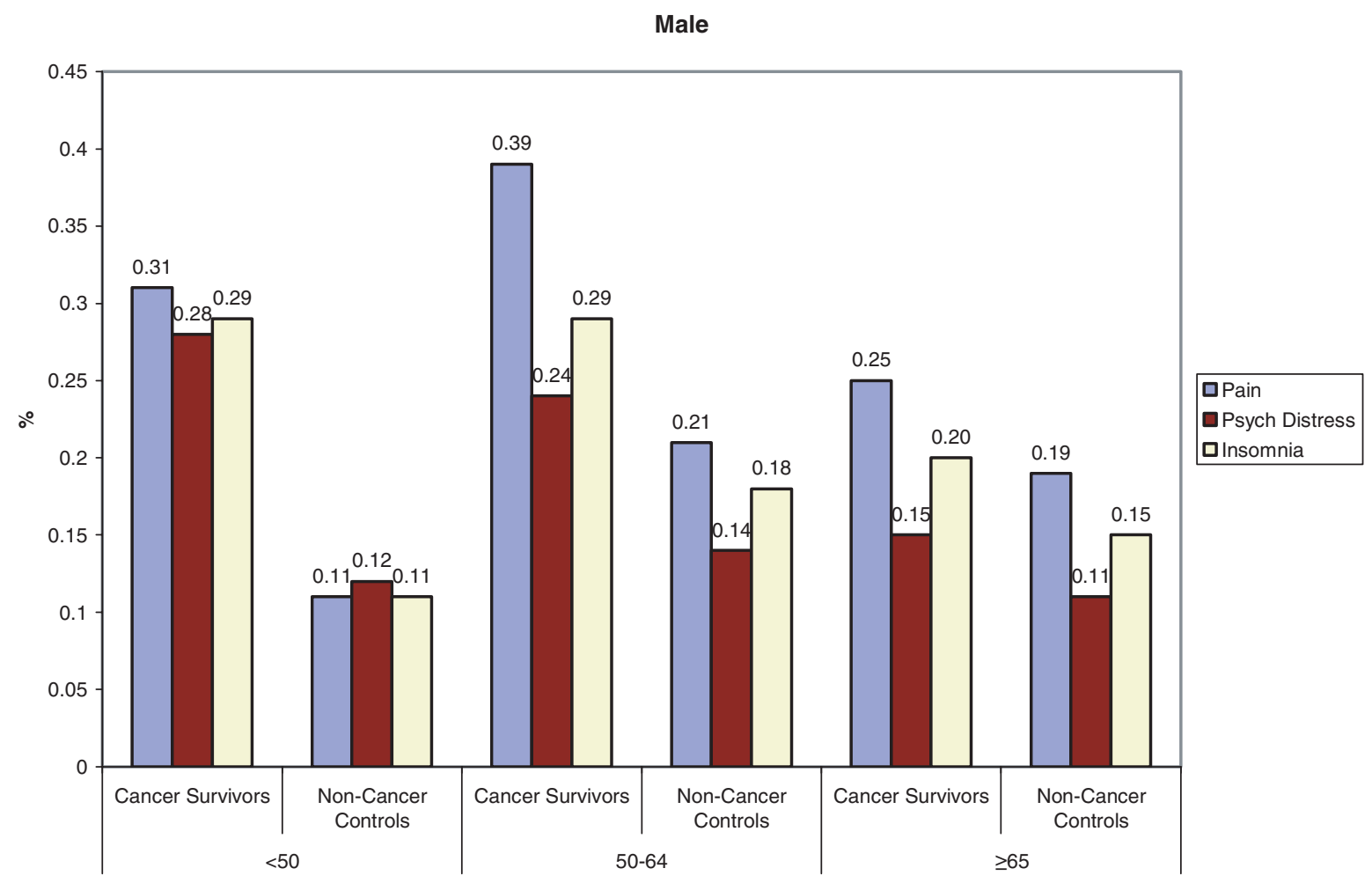

Female

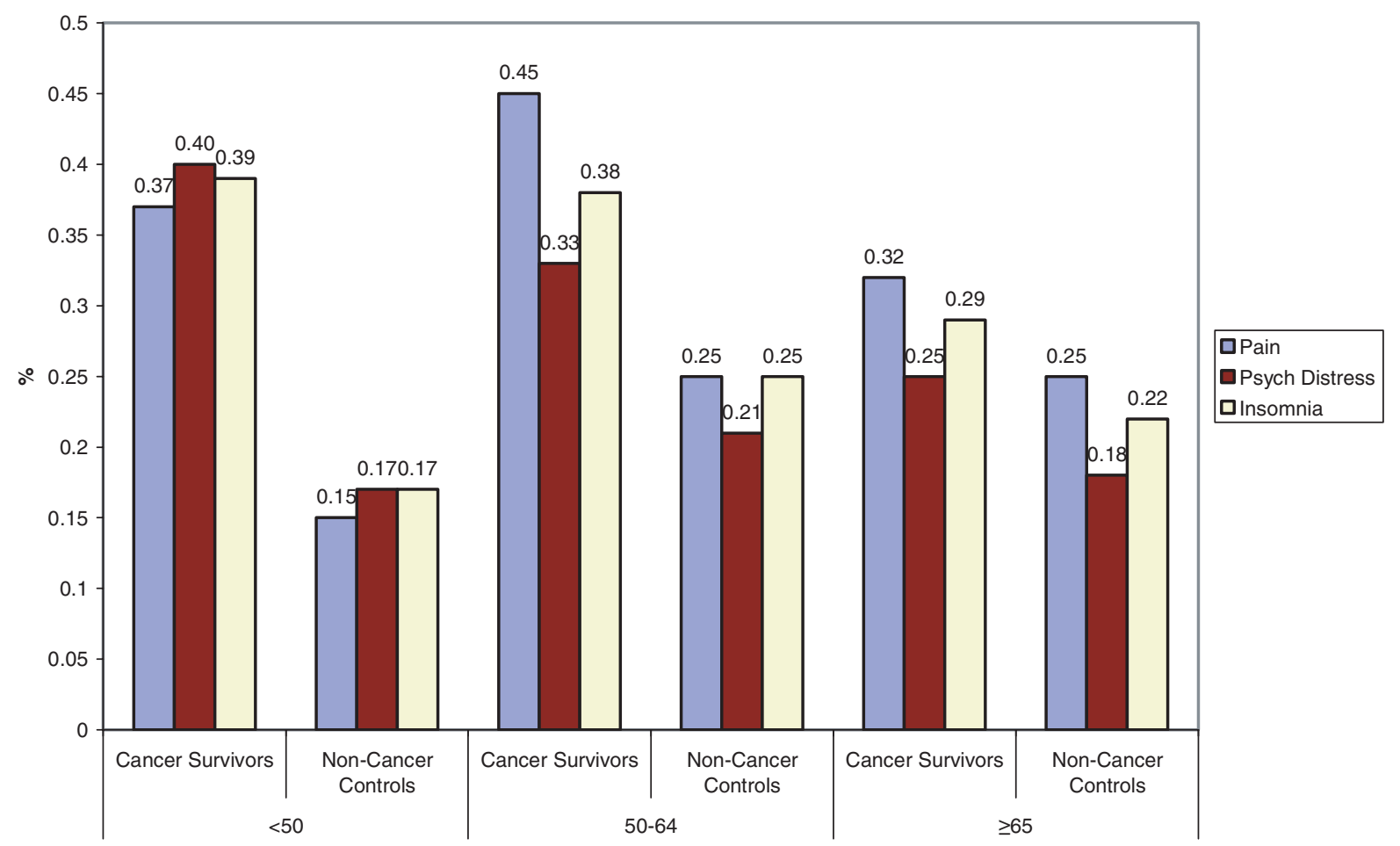

Figure 1. Probability of ongoing symptoms stratified by cancer survivorship, age, and sex. The final model included cancer survivorship, age, sex, education, race/ethnicity, and income (see Table 2). Average prediction was based on this model stratified by cancer survivor status, age, and sex. 
Table 3. Comorbidity and Increased Symptom Burden Among Cancer Survivors

\begin{tabular}{|c|c|c|c|c|c|c|}
\hline \multirow[b]{2}{*}{ Comorbidities* (n) $^{*}$} & \multicolumn{2}{|c|}{ Pain } & \multicolumn{2}{|c|}{ Psychological Distress } & \multicolumn{2}{|c|}{ Insomnia } \\
\hline & Control & CS & Control & CS & Control & CS \\
\hline 0 & $1(1.00-1.00)$ & $2.23(1.73-2.86)$ & $1(1.00-1.00)$ & $2.37(1.86-3.00)$ & $1(1.00-1.00)$ & $2.38(1.86-3.03)$ \\
\hline 1 & $7.44(6.67-8.29)$ & $10.02(8.08-12.43)$ & $3.37(3.08-3.70)$ & $4.57(3.71-5.61)$ & $3.52(3.21-3.86)$ & $4.54(3.64-5.66)$ \\
\hline 2 & $15.44(13.33-17.87)$ & $17.39(12.95-23.35)$ & $5.62(4.90-6.43)$ & $7.38(5.46-9.99)$ & $6.38(5.57-7.30)$ & $7.71(5.60-10.60)$ \\
\hline $3+$ & $27.34(22.49-33.23)$ & $35.56(25.34-49.91)$ & $11.26(9.37-13.54)$ & $15.12(11.08-20.64)$ & $9.92(8.36-11.78)$ & $10.25(7.40-14.21)$ \\
\hline
\end{tabular}

Models included comorbidity and cancer survivor status as interaction terms and were controlled for age, sex, education, race/ethnicity, and income. Data presented as odds ratios ( $95 \%$ confidence intervals).

${ }^{*}$ Comorbidities were measured by the number of health conditions causing functional limitations.

CS, cancer survivors.

tom estimation as illustrated by our study and others. ${ }^{23,24}$

We found a substantial symptom burden among cancer survivors compared with general population controls, with the greatest difference seen in patients younger than 50. Previous literature has found that younger survivors were more likely to report psychosocial problems and use mental health services compared with older survivors, ${ }^{24,25}$ and our study helps quantify this magnitude by comparing cancer survivors with the general population in the specific age categories. A number of reasons can account for why symptom burden may be greater among younger survivors. (1) Recent findings of age-related changes in the endogenous opioids $^{26}$ and serotonin ${ }^{27}$ systems may confer protection against perceived distress resulting from an age-related increase in physical problems. (2) Younger cancer survivors are more likely to choose definitive (eg, surgery) or systemic (eg, chemotherapy) cancer therapies, ${ }^{28}$ which are associated with greater side effects and long-term late effects. (3) As result of their other medical problems, older people may have developed coping strategies that can better mitigate the impact of cancer diagnosis and treatment. ${ }^{29}$ In contrast, younger people are often struck by a cancer diagnosis when they are in a state of "perfect health." A potentially life-threatening illness and intensive treatment regimen can interfere substantially with younger survivors' so-

Table 4. Symptom Burden by Cancer Type and Time Since Cancer Diagnosis, Adjusted for Age, Education, Race/ Ethnicity, and Income

\begin{tabular}{lccc}
\hline & Pain & Psychological Distress & Insomnia \\
\hline $\begin{array}{l}\text { Cancer type* } \\
\text { Breast (reference) }\end{array}$ & 1.00 & & 1.00 \\
Cervix & $1.10(0.71-1.71)$ & $1.86(1.17-2.96) \ddagger$ & 1.00 \\
Uterine & $1.22(0.75-1.99)$ & $2.08(1.25-3.46) \ddagger$ & $1.63(1.05-2.53) \dagger$ \\
Prostate & $1.06(0.68-1.63)$ & $0.82(0.47-1.41)$ & $1.63(1.00-2.65) \dagger$ \\
Colorectal & $0.95(0.59-1.53)$ & $1.00(0.58-1.71)$ & $0.58(0.36-0.95) \dagger$ \\
Melanoma & $0.87(0.52-1.46)$ & $0.92(0.50-1.68)$ & $0.77(0.47-1.27)$ \\
Multiple & $1.82(1.21-2.74) \dagger$ & $2.44(1.56-3.80) \ddagger$ & $0.62(0.35-1.09)$ \\
Other & $1.13(0.83-1.55)$ & $1.45(1.02-2.07) \dagger$ & $1.13(0.74-1.74)$ \\
Time since diagnosis & & & $0.91(0.66-1.26)$ \\
0 to 1 year (reference) & 1.00 & 1.00 & 1.00 \\
2 to 5 years & $0.83(0.61-1.13)$ & $0.76(0.55-1.06)$ & $0.66(0.48-0.90) \neq$ \\
6 to 10 years & $0.99(0.70-1.40)$ & $0.98(0.70-1.36)$ & $0.63(0.44-0.90) \neq$ \\
11 years or greater & $1.06(0.78-1.45)$ & $0.49-1.04)$ & $0.72(0.52-0.98) \dagger$ \\
\hline
\end{tabular}

Analysis included only cancer survivors. Data presented as odds ratios (95\% confidence intervals).

*Because several cancer types are sex specific, the models did not include sex as a covariate.

$+P<.05$.

$\ddagger P<.01$. 
cial and vocational needs, thus provoking greater perceived symptom frequency, severity, and bother. ${ }^{24}$ Further research is needed to better understand this relationship.

Our study demonstrated the impact of comorbidities in the context of cancer on symptom burden. Symptom burden increases drastically with the number of comorbidities. As the population ages, both cancer and other comorbidities increase; thus, how to research and deliver clinical care in the context of multiple competing health conditions becomes essential in effectively addressing the symptom burden among cancer survivors and those who suffer from other chronic diseases. Yancik et $\mathrm{a}^{28}$ has shown that comorbidity is an independent predictor of mortality among elderly breast cancer survivors controlled for age and cancer stages. Our study also suggested that those with comorbidities are the ones who bear the greatest symptom burden. Our analyses of time since diagnosis suggest that the burden of pain and psychological distress is persistent, without substantial decrease over time.

Our study also sheds light on the racial influences on symptom distress. Adjusting for socioeconomic status, we found that Hispanics and blacks had lower rates of ongoing symptom complaints compared with non-Hispanic whites, which seemed to differ from what is described in chronic pain literature. ${ }^{30,31}$ However, it is uncertain whether the lower rates are true or are caused by under-reporting related to communication and cultural barriers. ${ }^{32}$ Future research is needed to help uncover the different norms of cultural adaptation to symptom distress so effective communication, diagnosis, and treatment of these clinical problems can occur in health care settings.

The findings of this study need to be considered in light of its limitations. First, cancer survivors were identified through self-report. Cancer patients have been found to under-report histories of cancer diagnosis in an interview compared with medical records or tumor registries, ${ }^{33}$ which would have led to misclassification of cancer survivors as controls in our analysis. Second, the symptom burden in this study was also measured by self-report using simple questions. It captured an person's perceived ongoing symptom complaints but did not offer detailed information on severity, frequency, and interference. Future studies are needed to better understand how these salient features of symptom burden vary among cancer survivors and other appropriate populations. Third, our study attempted to provide an overview of symptom burden among US cancer survivors by collapsing heterogeneous cancer types into one broad category, as illustrated by our exploratory analysis; certain types of tumors may have higher symptom burden and future studies need to understand how symptoms relate to specific cancer or cancer specific treatments. Fourth, the NHIS database does not contain relevant clinical cancer staging or treatment information, which limited our ability to explore how these clinical factors contribute to symptom burden of cancer. Furthermore, the survey only included noninstitutionalized adults and so the results cannot be generalized to the pediatric or institutionalized adult population.

The strength of this study lies in the use of existing population-based NHIS data to provide an overview of symptom burden among cancer survivors with reference to the general population. The size of database allowed us to examine the intriguing interactions among cancer, age, and comorbidity. Our findings offer important implications. The substantial symptom burden experienced by cancer survivors in the community sample highlights that appropriate symptom assessment and management need to occur beyond the immediate treatment phase and major cancer centers. As medical science reduces the mortality rate for cancer and other diseases, increased resources need to be directed at addressing symptom control in cancer and other chronic illnesses. This is further emphasized by the fact that people often turn to non-evidence-based complementary therapies because of dissatisfaction with the efficacy of conventional treatments. ${ }^{34}$ To eliminate cancer suffering, multidisciplinary research and clinical efforts will be required to increase our understanding of the etiology of symptom burden, evaluate clinic therapeutics, and disseminate effective therapies to cancer survivors. Because primary care physicians play central role in coordinating care and managing chronic illnesses, only by engaging primary care physicians in the design, implementation, and dissemination of research will we generate scientifically and clinically meaningful solutions that benefit millions of cancer survivors.

\section{References}

1. Jemal A, Clegg LX, Ward E, et al. Annual report to the nation on the status of cancer, 1975-2001, with a 
special feature regarding survival. Cancer 2004;101: 3-27.

2. Yabroff K, Lawrence W, Clauser S, Davis W, Brown M. Burden of illness in cancer survivors: findings from a population-based national sample. J Natl Cancer Inst 2004;96:1322-30.

3. Goudas LC, Bloch R, Gialeli-Goudas M, Lau J, Carr DB. The epidemiology of cancer pain. Cancer Invest 2005;23:182-90.

4. Cleeland CS, Gonin R, Hatfield AK, et al. Pain and its treatment in outpatients with metastatic cancer. N Engl J Med 1994;330(9):592-6.

5. Portenoy R, Kornblith A, Wong G, et al. Pain in ovarian cancer patients. Prevalence, characteristics, and associated symptoms. Cancer 1994;74:907-15.

6. Ciaramella A, Poli P. Assessment of depression among cancer patients: the role of pain, cancer type, and treatment. Psychooncology 2001;10:156-65.

7. Rao A, Cohen HJ. Symptom management in the elderly cancer patient: fatigue, pain, and depression. J Nat Cancer Inst Monographs 2004;32:150-7.

8. Davidson JR, MacLean AW, Brundage MD, Schulze K. Sleep disturbance in cancer patients. Soc Sci Med 2002;54:1309-21.

9. Patrick DL, Ferketich SL, Frame PS, et al. National Institutes of Health State-of-the-Science conference statement: symptom management in cancer: pain, depression, and fatigue, July 15-17, 2002. J Natl Cancer Inst 2003;95:1110-7.

10. Massie MJ. Prevalence of depression in patients with cancer. J Nat Cancer Inst Monographs 2004; 32:57-71.

11. Hosaka T, Aoki T. Depression among cancer patients. Psychiatry Clin Neurosci 1996;50:309-12.

12. Grond S, Zech D, Diefenbach C, Bischoff A. Prevalence and pattern of symptoms in patients with cancer pain: a prospective evaluation of 1635 cancer patients referred to a pain clinic. J Pain Symptom Manage 1994;9:372-82.

13. Savard J, Morin CM. Insomnia in the context of cancer: a review of a neglected problem. J Clin Oncol 2001;19:895-908.

14. Gureje O, Von Korff M, Simon GE, Gater R. Persistent pain and well-being: a World Health Organization study in primary care. JAMA 1998; 280:147-51.

15. Thase ME. Correlates and consequences of chronic insomnia. Gen Hosp Psychiatry 2005;27:100-12.

16. Goyal TM, Idler EL, Krause TJ, Contrada RJ. Quality of life following cardiac surgery: impact of the severity and course of depressive symptoms. Psychosom Med 2005;67:759-65.
17. National Center for Health Statistics. National Health Interview Survey 2002: Machine Readable Data File and Documentation. Hyattsville (MD): Department of Health and Human Services, Centers for Disease Control and Prevention; 2002.

18. Centers for Disease Control and Prevention, $\mathrm{Na}$ tional Center for Health Statistics. National Health Interview Survey. Available at http://www.cdc.gov/ nchs/nhis.htm. Accessed 14 June 2006.

19. Kroenke K. Studying symptoms: sampling and measurement issues. Ann Intern Med 2001;134(9 Pt 2): 844-53.

20. American Statistical Association. Section on survey research methods: summary of survey analysis software. Available at: http://www.fas.harvard.edu/ $\sim$ stats/survey-soft/survey-soft.html. Accessed 12 Feb 2006.

21. Barnes PM, Powell-Griner E, McFann K, Nahin RL. Complementary and alternative medicine use among adults: United States, 2002. Adv Data 2004; (343):1-19.

22. Rowland JH, Yancik R. Cancer survivorship: the interface of aging, comorbidity, and quality care. J Natl Cancer Inst 2006;98:504-5.

23. Miaskowski C, Dodd M, Lee K. Symptom clusters: the new frontier in symptom management research. J Nat Cancer Inst Monographs 2004;32:17-21.

24. Baker F, Denniston M, Smith T, West MM. Adult cancer survivors: How are they faring? Cancer 2005; 104(11 Suppl):2565-76.

25. Hewitt M, Rowland JH. Mental health service use among adult cancer survivors: analyses of the $\mathrm{Na}$ tional Health Interview Survey. J Clin Oncol 2002; 20:4581-90.

26. Zubieta JK, Dannals RF, Frost JJ. Gender and age influences on human brain mu-opioid receptor binding measured by PET. Am J Psychiatry 1999;156: $842-8$.

27. Newberg AB, Amsterdam JD, Wintering N, et al. 123I-ADAM binding to serotonin transporters in patients with major depression and healthy controls: a preliminary study. J Nucl Med 2005;46:973-7.

28. Yancik R, Wesley MN, Ries LA, Havlik RJ, Edwards BK, Yates JW. Effect of age and comorbidity in postmenopausal breast cancer patients aged 55 years and older. JAMA 2001;285:885-92.

29. Cassileth BR, Lusk EJ, Strouse TB, et al. Psychosocial status in chronic illness. A comparative analysis of six diagnostic groups. N Engl J Med 1984;311: $506-11$.

30. Bates MS. Biocultural dimensions of chronic pain: implications for treatment of multi-ethnic populations. Albany (NY): State University of New York Press; 1996.

31. Riley JL 3rd, Wade JB, Myers CD, Sheffield D, 
Papas RK, Price DD. Racial/ethnic differences in the experience of chronic pain. Pain 2002;100:291-8.

32. Cleeland CS, Gonin R, Baez L, Loehrer P, Pandya KJ. Pain and treatment of pain in minority patients with cancer. The Eastern Cooperative Oncology Group Minority Outpatient Pain Study. Ann Intern Med 1997;127:813-6.

33. Desai MM, Bruce ML, Desai RA, Druss BG. Valid- ity of self-reported cancer history: a comparison of health interview data and cancer registry records. Am J Epidemiol 2001;153:299-306.

34. Eisenberg DM, Kessler RC, Van Rompay MI, et al. Perceptions about complementary therapies relative to conventional therapies among adults who use both: results from a national survey. Ann Intern Med 2001;135:344-51. 